\title{
MEROMORPHIC FUNCTIONS ON CERTAIN RIEMANN SURFACES WITH SMALL BOUNDARY
}

\author{
PENTTI JÄRVI
}

\section{Introduction}

In this paper we study meromorphic functions on certain Riemann surfaces with ,removable" ideal boundary, in particular functions which admit continuous extensions to the ideal boundary ( $M C$-functions). Our purpose is to generalize or extend certain results of Heins ([3], [4]), L. Myrberg ([7]), Ozawa ([9]) and Royden ([10]). In particular, two composition theorems, originally due to Heins ([3, p. 304], [4]), will be unified to statements on $M C$-functions on Riemann surfaces satisfying the absolute $A B$-maximum principle in the sense of Royden. An essential feature in the considerations involved is the coincidence of the class $M C$ with the class of constants and of meromorphic functions of bounded valence. Motivated by this fact, we will, in Chapter 2, make an attempt to characterize those Riemann surfaces for which these two classes coincide. This effort leads also to further extensions of some results obtained in Chapter 1.

The author wishes to thank Professor K. I. Virtanen for valuable comments.

\section{Riemann surfaces with the absolute $A B$-maximum principle}

1.1. Let $W$ be an open Riemann surface and iet $V$ be a subregion of $W$ with compact (possibly empty) relative boundary $\partial V$. Let $\beta$ denote the KerékjártóStoïlow ideal boundary of $W$ and $\beta_{V}$ the relative Kerékjártó-Stoïlow ideal boundary of $V$ (see [12, p. 366]). Denote by $A(V)$ or $A(\bar{V})$ the class of analytic functions on $V$ or $\bar{V}=V \cup \partial V$ and by $M(V)$ or $M(\bar{V})$ the class of meromorphic functions on $V$ or $\bar{V}$, respectively. The class constituted by the bounded functions in $A(V)$ or $A(\bar{V})$ is denoted by $A B(V)$ or $A B(\bar{V})$, respectively. The subclass of $A(V)$ (resp. $M(V)$ ) consisting of functions which have a finite (resp. finite or infinite) limit at every relative ideal boundary element is denoted by $A C(V)$ (resp. $M C(V)$ ). The classes $A C(\bar{V})$ and $M C(\bar{V})$ are defined similarly. Whenever $f$ is a function of class $A C$ or $M C$, we let $f^{*}$ denote the extension of $f$ to the (relative) ideal boundary. 
We say that $W$ satisfies the absolute $A B$-maximum principle ([10]), and denote $W \in \mathscr{M}_{B}$ if

$$
\sup \{|f(p)| \mid p \in \bar{V}\}=\max \{|f(p)| \mid p \in \partial V\}
$$

for every subregion $V$ of $W$ with compact $\partial V$ and for every $f \in A B(\bar{V})$. Further, $W$ is said to belong to the class $\mathscr{D}_{B}([9])$ if, for every subregion $V$ with compact $\partial V$, the cluster set $\mathrm{C} 1\left(f ; \beta_{V}\right)$ of every $f \in A B(\bar{V})$ attached to the relative ideal boundary $\beta_{V}$ is a totally disconnected subset of $C$, the complex plane. Clearly, $A B(\bar{V}) \subset$ $A C(\bar{V})$ whenever $W$ belongs to $\mathscr{D}_{B}$. As usual, $\mathcal{O}_{A B}$ denotes the class of all Riemann surfaces on which there exists no nonconstant bounded analytic function. The following theorem reveals the intimate relations between these three classes. To prove it, and in certain other connections as well, we need some characterizations of the properness of an analytic mapping; proofs can be found in [5, pp. 7-8].

Lemma 1. Let $W$ be a disjoint union of at most a countable number of Riemann surfaces, and let $G$ be a domain in the Riemann sphere $\hat{C}=C \cup\{\infty\}$. Suppose that $f: W \rightarrow G$ is analytic. Then the following statcments are equivalent:

(1) $f$ is proper.

(2) The valence function $v_{f}(z)=\sum_{f(p)=z} n(p ; f)$, where $n(p ; f)$ denotes the multiplicity of $f$ at $p$, is finite and constant on $G$.

(3) Given any sequence $\left(p_{n}\right)$ in $W$ such that $\left\{n \in N \mid p_{n} \in K\right\}$ is finite for every compact set $K \subset W$, the same is true of $\left(f\left(p_{n}\right)\right)$ and every compact set $K^{\prime}$ in $G$. Moreover, if any of the above conditions is satisfied, then the number of the components of $W$ is finite, and each of them is mapped properly on $G$.

Theorem 1. For Riemann surfaces of finite genus the classes $\mathscr{M}_{B}, \mathscr{D}_{B}$ and $\mathcal{O}_{A B}$ coincide. For surfaces of infinite genus we have

$$
\mathscr{M}_{B}=\mathscr{D}_{B} \subsetneq \mathcal{O}_{A B} \text {. }
$$

Proof. Surfaces of finite genus in $\mathcal{O}_{A B}$ are complements on closed surfaces of sets of the class $N_{B}$ ([12, p. 137]; for the definitions and basic properties of the function-theoretic null-classes $N_{B}, N_{D}$ and $N_{S B}$ we refer to [1] or [12, Chapter 2]). So it follows immediately that $\mathscr{M}_{B}=\mathcal{O}_{A B}=\mathscr{D}_{B}$ for these surfaces. As concerns surfaces of infinite genus, the relation $\mathscr{D}_{B} \subset \mathscr{M}_{B}$ was proved in [9, Theorem 1]. Moreover, it is trivial that $\mathscr{M}_{B} \subset \mathcal{O}_{A B}$ generally, and the strictness of this inclusion is shown by P. J. Myrberg's example (see e.g. [12, p. 53]). It remains to prove $\mathscr{M}_{B} \subset \mathscr{D}_{B}$.

Let $W \in \mathscr{M}_{B}$, and let $V$ be a subregion of $W$ with compact $\partial V$. Passing to a suitable subset of $V$, we may assume that $\partial V$ consists of a finite number of piecewise analytic closed curves. Let $f \in A B(\bar{V})$ be nonconstant. Assuming that $z \in C \backslash f(\partial V)$, the index of $z$ is defined by

$$
i(z)=(2 \pi)^{-1} \int_{\partial V} d \arg (f(p)-z) .
$$


With suitable interpretation (see [10]), $i(z)$, as well as the valence $v(z)$ of $f$ at $z$ with respect to $\bar{V}$, can be defined also for $z \in f(\partial V)$ and expressly in such a way that $\delta(z)=i(z)-v(z)=0$ everywhere in $C$ whenever $V$ is relatively compact.

The fundamental result of [10] now states that $f$ has bounded valence and actually $\delta(z) \geqq 0$ for all $z \in \boldsymbol{C}$; moreover, the closed set $E=\{z \in \boldsymbol{C} \mid \delta(z)>0\}$ is of class $N_{B}$. We proceed to show that $\mathrm{Cl}\left(f ; \beta_{V}\right) \subset E$.

Since $\delta$ (and $E$ ) is unaltered by the removal from $V$ of a compact set with nice boundary, we may assume that $f(\partial V) \cap E=\emptyset$. Let $z \in f(\partial V)$. We assert that there exists a closed neighborhood $N$ of $z$ such that $f^{-1}(\overline{f(V)} \cap N) \cap \bar{V}$ is compact. Indeed, since $f$ is analytic on $\bar{V}$, we may replace $V$ by a larger region $V^{\prime}$ with nice boundary such that $\bar{V}^{\prime} \backslash V$ is compact and $z \notin f\left(\partial V^{\prime}\right)$. Moreover, if $G_{z}$ is the component of $C \backslash f\left(\partial V^{\prime}\right)$ which contains $z$, then by virtue of Lemma $1 f \mid f^{-1}\left(G_{z} \backslash E\right) \cap V^{\prime}$ is a proper mapping onto $G_{z} \backslash E$. Thus $f^{-1}(N) \cap V^{\prime}$ is compact whenever $N$ is e.g. a closed disc in $G_{z} \backslash E$ with $z$ in its interior. Since $f^{-1}(N) \cap \bar{V}=f^{-1}(\overline{f(V)} \cap N) \cap \bar{V}$, the assertion follows. One consequence of the assertion is the compactness of $f^{-1}(f(\partial V)) \cap \bar{V}$. Thus $f^{-1}(C \backslash f(\partial V)) \cap V$ consists of a finite number of components with compact relative boundaries (Lemma 1).

Now let $p \in \beta_{V}$, and denote by $U$ the component of $f^{-1}(C \backslash(f(\partial V) \cup E)) \cap V$ such that $p \in \beta_{U}$. It follows from Lemma 1 that $f \mid U$ is a proper mapping onto $G$, a component of $C \backslash(f(\partial V) \cup E)$. By the same lemma, the cluster set $\mathrm{C} 1(f ; p)$ of $f$ at $p$ is contained in $\partial G$. But the assertion proved above implies that $\mathrm{C} 1(f ; p) \cap f(\partial V)=\emptyset$, so that only the case $\mathrm{Cl}(f ; p) \subset E$ is feasible. The proof is complete.

Let $W$ be a Riemann surface, and let $V$ be a subregion of $W$. Denote by $B V(V)$ or $B V(\bar{V})$ the class of constants and of meromorphic functions of bounded valence on $V$ or $\bar{V}$, respectively. For a nonconstant function $f \in B V(W)$ the deficiency set of $f$ is defined by

$$
D_{f}=\left\{z \in \hat{\boldsymbol{C}} \mid v_{f}(z)<\max _{z \in \hat{C}} v_{f}(z)\right\} .
$$

Further denote by $M D^{*}(V)\left(M D^{*}(\bar{V})\right)$ the class of meromorphic functions on $V(\bar{V})$ with finite spherical Dirichlet integrals, and let $\mathcal{O}_{M D^{*}}$ denote the class of Riemann surfaces which tolerate no nonconstant $M D^{*}$-function. Recall that the spherical Dirichlet integral of a meromorphic function $f$ on $W$ is defined by

$$
\iint_{W} \frac{1}{\left(1+|f(p)|^{2}\right)^{2}} d f \wedge * d \bar{f} .
$$

We are now ready to state the following corollary; cf. [7, Satz 3.1, Satz 3.2 and Satz 3.3], [9, Theorem 3] and [12, Theorem VI. 3 C and Theorem VI. 3 D] (note that $\mathcal{O}_{A^{\circ} B} \subset \mathscr{M}_{B}$ by [12, Theorem VI. $3 \mathrm{~A}$ and Theorem VI. $\left.\left.1 \mathrm{I}\right]\right)$.

Theorem 2. Let $W \in \mathscr{M}_{B}$, and let $V$ be a subregion of $W$ with compact boundary. Then

$$
A B(\bar{V})=A C(\bar{V}) \quad \text { and } \quad M C(\bar{V})=B V(\bar{V})=M D^{*}(\bar{V}) .
$$


In particular,

$$
M C(W)=B V(W)=M D^{*}(W),
$$

and $D_{f} \in N_{B}$ for every nonconstant $f \in B V(W)$. Moreover, if $f \in M(\bar{V}) \backslash M C(\bar{V})$, then $f$ assumes every value in $\hat{C}$ infinitely often except for a set of values with compact parts in $N_{B}$.

Proof. The first equality is an immediate consequence of Theorem 1. Suppose that $f \in B V(\bar{V})$ is nonconstant. As in the proof of [5, Lemma 6], we can find a linear fractional mapping $\psi$ such that $\psi \circ f$ is bounded off a compact set $K \subset \bar{V}$. By Theorem 1, $\psi \circ f$ has a limit at every element of $\beta_{V}$, and so has $f$. Thus $B V(\bar{V}) \subset$ $M C(\bar{V})$. A similar reasoning justifies the assertion concerning $D_{f}$ for $f \in B V(W)$. Indeed, the number of the noncompact components of $W \backslash K$ is finite, and the proof of Theorem 1 combined with [12, Theorem VI. $1 \mathrm{~L}]$ implies that $(\psi \circ f)^{*}(\beta)$ is of class $N_{B}$. Hence $f^{*}(\beta) \in N_{B}$ as well. The assertion now follows from Lemma 1 .

Next assume that $f \in M C(\bar{V})$ is nonconstant. Let $p \in \beta_{V}$. For a suitable linear fractional mapping $\varphi, \varphi \circ f^{*}$ is bounded in an open neighborhood $U_{p}$ of $p$. By the proof of Theorem 1, $\left(\varphi \circ f^{*}\right) \mid U_{p} \backslash \beta_{V}$ has bounded valence. Hence the same holds for $f^{*} \mid U_{p} \backslash \beta_{V}$ as well. Thus we can find for every $p \in \bar{V} \cup \beta_{V}$ an open neighborhood $U_{p}$ such that $f^{*} \mid U_{p} \backslash \beta_{V}$ has bounded valence. By compactness $f$ has bounded valence, too. We infer that $M C(\bar{V}) \subset M D^{*}(\bar{V})$.

To prove the remaining inclusion $M D^{*}(\bar{V}) \subset B V(\bar{V})$, suppose that $f \in M D^{*}(\bar{V})$ is nonconstant but fails to have bounded valence. Given a relative regular exhaustion $\left(R_{n}\right)$ of $\bar{V}$, let $E_{n}=\hat{\boldsymbol{C}} \backslash f\left(V \backslash \bar{R}_{n}\right), n=1,2, \ldots$ Then $E_{n}$ is a closed set of class $N_{B}$ for each $n$, for otherwise we could find a nonconstant bounded analytic function $g$ on $\hat{\boldsymbol{C}} \backslash E_{n}$, in which case $(g \circ f) \mid \bigvee \backslash \bar{R}_{n}$ would also be bounded. But this state of affairs contradicts the relation $f \notin B V(\bar{V})$ by the proof of Theorem 1. Clearly $\left\{z \in \hat{\boldsymbol{C}} \mid v_{f}(z)<\infty\right\} \subset \bigcup_{n=1}^{\infty} E_{n}$, and $E_{n} \in N_{B}$ implies area $\left(E_{n}\right)=0$ for each $n$. Accordingly, $f \notin M D^{*}(\bar{V})$. The desired contradiction has been reached. The same reasoning proves the last statement of the theorem in view of [12, Theorem VI. $1 \mathrm{~L}]$.

Let $P$ be a class of closed sets in $\hat{C}$. We denote by $\mathscr{D}(P)$ the class of all Riemann surfaces which tolerate a nonconstant $B V$-function with the deficiency set in $P$. The following theorem provides a description of Riemann surfaces with the absolute $A B$-maximum principle up to the quite strange class $\mathcal{O}_{M D^{*}}$.

Theorem 3. $\mathscr{M}_{B} \backslash \mathcal{O}_{M D^{*}}=\mathscr{D}\left(N_{B}\right)$.

Proof. Suppose that $W \in \mathscr{M}_{B} \backslash \mathcal{O}_{M D^{*}}$. By Theorem 2 there exists a nonconstant function $f \in M C(W)$, and by the same theorem $D_{f} \in N_{B}$, i.e. $W \in \mathscr{D}\left(N_{B}\right)$.

Now let $W \in \mathscr{D}\left(N_{B}\right)$, and let $f_{0}$ be a nonconstant element in $B V(W)$ with $D_{f_{0}}$ in $N_{B}$. We may assume that $D_{f_{0}} \subset C$. Let $V$ be a subregion of $W$ whose relative boundary $\partial V$ consists of a finite number of piecewise analytic closed curves. By modifying slightly $\partial V$, we obtain $f_{0}(\partial V) \cap D_{f_{0}}=\emptyset$. Let $G$ be a component of 
$\hat{\boldsymbol{C}} \backslash f_{0}(\partial V)$ such that $G \cap D_{f_{0}} \neq \emptyset$. Denoting by $\beta$ the ideal boundary of $W$, we have $\mathrm{C} 1\left(f_{0} ; \beta\right) \subset D_{f_{0}}$ (Lemma 1). Again by Lemma $1, v_{f_{0} \mid V}$ is finite and constant $(>0)$ on $G \backslash D_{f_{0}}$.

Let $U$ be a relatively noncompact component of $f_{0}^{-1}(G)$. Then $U \backslash f_{0}^{-1}\left(D_{f_{0}}\right)$ is mapped by $f_{0}$ properly on $G \backslash D_{f_{0}}$ (Lemma 1$)$. So there exists a positive integer $n$ such that $v_{f_{0} \mid U \backslash f_{0}^{-1}\left(D_{f_{0}}\right)}(z)=n$ for every $z \in G \backslash D_{f_{0}}$. Now suppose that $f \in A B(\bar{V})$. By an argument borrowed from the theory of compact Riemann surfaces, it can be shown that $f$ satisfies on $U \backslash f_{0}^{-1}\left(D_{f_{0}}\right)$ an identity

$$
f^{n}+\sum_{i=1}^{n}\left(a_{i} \circ f_{0}\right) f^{n-i}=0,
$$

where $a_{1}, \ldots, a_{n}$ are bounded analytic functions on $G \backslash D_{f_{0}}$. But $D_{f_{0}}$ is of class $N_{B}$, so that we may regard the functions $a_{i}$ as defined all over $G$. Moreover, it is a standard consequence of the connectedness of $U \backslash f_{0}^{-1}\left(D_{f_{0}}\right)$ that

$$
P(z, w)=w^{n}+\sum_{i=1}^{n} a_{i}(z) w^{n-i}=Q(z, w)^{m^{\prime}},
$$

where $Q(z, w)$ is an irreducible polynomial over the $\operatorname{ring} M(G)$ of degree $m=n / m^{\prime}$.

Denote by $\widetilde{G}$ the (connected) Riemann surface of the relation $Q(z, w)=0$, $z \in G$, i.e. the totality of pairs $\left(z, w_{z}\right)$, where $z \in G$ and $w_{z}$ is a function element with center $z$ and associated with the equation $Q(z, w)=0$. The center function $c$ : $\left(z, w_{z}\right) \mapsto z$ and the value function $v:\left(z, w_{z}\right) \mapsto w_{z}(z)$ are analytic on $\widetilde{G}$.

Denote by $\varphi$ the analytic mapping $U \rightarrow \widetilde{G}$,

$$
p \mapsto\left(f_{0}(p),\left(f \circ f_{0}^{-1}\right)_{f_{0}(p)}\right),
$$

where the branch of $f_{0}^{-1}$ is chosen in such a way that $f_{0}^{-1}\left(f_{0}(p)\right)=p$ (the points $p \in U$ at which $f_{0}$ fails to be a local homeomorphism are removable singularities by continuity and by Riemann's theorem on isolated singularities). We have immediately $f_{0}=c \circ \varphi, f=v \circ \varphi$.

We will show that $\varphi$ admits a continuous extension $\varphi^{*}$ to $U \cup \beta_{U}$ with $\varphi^{*}\left(\beta_{U}\right) \subset c^{-1}\left(G \cap D_{f_{0}}\right)$. So let $q_{0} \in \mathrm{Cl}\left(\varphi ; \beta_{U}\right) \subset \tilde{G} \cup \beta^{\prime}$, where $\beta^{\prime}$ denotes the ideal boundary of $\tilde{G}$, and let $\left(p_{n}\right)$ be a sequence of points in $U$ such that $p_{n} \rightarrow p_{0}$ for some $p_{0} \in \beta_{U}$ and $\varphi\left(p_{n}\right) \rightarrow q_{0}$. Assume that $q_{0} \in \widetilde{G} \backslash c^{-1}\left(G \cap D_{f_{0}}\right)$. Then $(c \circ \varphi)\left(p_{n}\right) \rightarrow$ $c\left(q_{0}\right) \in G \backslash D_{f_{0}}$, which is impossible since $f_{0}\left(p_{n}\right) \rightarrow D_{f_{0}}$. Similarly, the assumption $q_{0} \in \beta^{\prime}$ leads to the contradictory result $f_{0}\left(p_{n}\right) \rightarrow \partial G$. Hence we have $q_{0} \in c^{-1}\left(G \cap D_{f_{0}}\right)$. Since $c^{-1}\left(G \cap D_{f_{0}}\right)$ is totally disconnected, $\mathrm{C} 1(\varphi ; p)$ reduces to a singleton for every $p \in \beta_{U}$, i.e. $\varphi$ admits a continuous extension $\varphi^{*}$ to $U \cup \beta_{U}$. Moreover, $\varphi^{*}(p) \in c^{-1}\left(G \cap D_{f_{0}}\right)$ for every $p \in \beta_{U}$.

Again let $\left(p_{n}\right)$ be a sequence of points in $U$ such that $p_{n} \rightarrow p_{0}$ for some $p_{0} \in \beta_{U}$. Then $\varphi\left(p_{n}\right) \rightarrow \varphi^{*}\left(p_{0}\right) \in c^{-1}\left(D_{f_{0}} \cap G\right)$, so that $(v \circ \varphi)\left(p_{n}\right) \rightarrow v\left(\varphi^{*}\left(p_{0}\right)\right) \in v\left(c^{-1}\left(D_{f_{0}} \cap G\right)\right)$, i.e. $\lim _{n \rightarrow \infty} f\left(p_{n}\right) \in v\left(c^{-1}\left(D_{f_{0}} \cap G\right)\right)$. Since $v\left(c^{-1}\left(D_{f_{0}} \cap G\right)\right)$ is totally disconnected (note that $c^{-1}\left(D_{f_{0}} \cap G\right)$ is compact by Lemma 1$)$ and $\beta_{V}$ is a finite union of sets of 
type $\beta_{U}, \mathrm{Cl}\left(f ; \beta_{V}\right)$ is totally disconnected as well. Thus $W \in \mathscr{D}_{B}$, and by Theorem 1 $W \in \mathscr{M}_{B}$, too.

1.2. We turn to the analogues of Heins' composition theorems. As concerns globally defined functions, we have

Theorem 4. Let $W$ be a Riemann surface of class $\mathscr{M}_{B}$. Then either

(a) $M C(W)=C$, in which case $W \in \mathcal{O}_{M D^{*}}$,

or

(b) $W \in \mathscr{D}\left(N_{B}\right)$, and $M C(W)$ is a field algebraically isomorphic to the field of rational functions on a compact Riemann surface $W^{\prime}$, which is uniquely determined up to a conformal equivalence. Moreover, the isomorphism is induced by an analytic mapping of $W$ into $W^{\prime}$.

Proof. If $W \in \mathscr{M}_{B} \cap \mathcal{O}_{M D^{*}}$, then by Theorem 2 every function in $M C(W)$ is constant. If, on the other hand, $W \in \mathscr{D}\left(N_{B}\right)$, we may apply the result of Heins ([4]; cf. also [5, Theorem 7]), for by Theorem $2 M C(W)=B V(W)$.

Again let $W \in \mathscr{M}_{B}$, and let $p_{0} \in \beta$, the ideal boundary of $W$. Suppose that there exists a subregion $V$ of $W$ which belongs to a determining sequence of $p_{0}$ and carries on its closure $\bar{V}$ a nonconstant bounded analytic function $f$. By Theorem $2, f$ admits a continuous extension $f^{*}$ to $\bar{V} \cup \beta_{V}$. Assume, as we may, that $z_{0}=$ $f^{*}\left(p_{0}\right) \notin f(\partial V)$. Denote, as in the proof of Theorem 1 , by $E\left(\in N_{B}\right)$ the set $\{z \in \boldsymbol{C} \mid \delta(z)=i(z)-v(z)>0\}$ and by $G$ the component of $\boldsymbol{C} \backslash f(\partial V)$ which contains $z_{0}$ (note that $z_{0} \in E$ as appears from the proof of Theorem 1 ). Let $\left(D_{j}\right)$ be a decreasing sequence of Jordan domains in $G$ such that $\partial D_{j} \cap E=\emptyset, j=1,2, \ldots$, and $\cap_{j=1}^{\infty} \bar{D}_{j}=\left\{z_{0}\right\}$. Denote by $U_{j}$ the component of $f^{-1}\left(D_{j} \backslash E\right)$ such that $p_{0} \in \beta_{U_{j}}$ and by $n_{j}$ the constant valence of $f \mid U_{j}$ on $D_{j} \backslash E, j=1,2, \ldots$ (cf. Lemma 1). Note, for future use, that (Int $\bar{U}_{j}$ ) is a determining sequence of $p_{0}$, i.e. $\bigcap_{j=1}^{\infty} \bar{U}_{j}=\emptyset$, and also that $\left(f^{*}\right)^{-1}\left(z_{0}\right)$ contains at most $i\left(z_{0}\right)$ points (in $\bar{V} \cup \beta_{V}$ ), for the number of the components of $f^{-1}\left(D_{j}\right)$ is at most $i\left(z_{0}\right)$ for each $j$. The integer

$$
n\left(p_{0} ; f\right)=n(f)=\lim _{j \rightarrow \infty} n_{j}
$$

is called the multiplicity or the local degree of $f$ at $p_{0}$ (cf. [3, p. 301] and [7, p. 8]). It is clearly independent of the choice of $\left(D_{j}\right)$. Furthermore, let

$$
n=\min \{n(f) \mid f \text { is an admitted function }\} .
$$

By the definition above, there exist a subregion $V_{0}$ of $W$ with compact $\partial V_{0}$ such that $p_{0} \in \beta_{V_{0}}$ and an analytic function $f_{0}: V_{0} \rightarrow D=\{z \in C|| z \mid<1\}$ such that $v_{f_{0}}(z)=n$ for all $z \in D$ except for a compact set $E_{0}$ of class $N_{B}$ and $f_{0}^{*}\left(\beta_{V_{0}}\right) \subset E_{0}$. With the notation before, we have (cf. [3, p. 304], [9, p. 751])

Theorem 5. Given $f \in M C\left(V_{0}\right)$, there exists a unique $g \in M(D)$ such that $f=g \circ f_{0}$. A fortiori, $M(D)$ is algebraically isomorphic to $M C\left(V_{0}\right)$; in particular, $g \mapsto g \circ f_{0}$ is an isomorphism of $A B(D)$ onto $A B\left(V_{0}\right)$. 
Proof. Let $f \in M C\left(V_{0}\right)$. As in the proof of Theorem 3, it can be shown that $f$ satisfies on $f_{0}^{-1}\left(D \backslash E_{0}\right)$ a relation

$$
f^{n}+\sum_{i=1}^{n}\left(a_{i} \circ f_{0}\right) f^{n-i}=0
$$

where $a_{1}, \ldots, a_{n}$ are meromorphic functions on $D \backslash E_{0}$. We will show that $a_{i}$ admits a meromorphic extension to $E_{0}$ for each $i$. Fix $z_{0} \in E_{0}$, and denote by $p_{1}, \ldots, p_{k}$ $(k \leqq n)$ the points of $\left(f_{0}^{*}\right)^{-1}\left(z_{0}\right)$. Since $f^{*}$ is continuous, there exists a neighborhood $U$ of $\left\{p_{1}, \ldots, p_{k}\right\}$ in $V_{0} \cup \beta_{V_{0}}$ such that $\hat{C} \backslash f^{*}(U)$ contains an open set. Hence there exists a linear fractional mapping $\varphi$ such that $f_{1}=\left(\varphi \circ f^{*}\right) \mid U$ is bounded. Now choose a neighborhood $G \subset D$ of $z_{0}$ in such a way that $\partial G \cap E=\emptyset$ and $\left(f_{0}^{*}\right)^{-1}(G) \subset U$. Then $f_{1}$ satisfies on $f_{0}^{-1}\left(G \backslash E_{0}\right)$ an identity

$$
f_{1}^{n}+\sum_{i=1}^{n}\left(b_{i} \circ f_{0}\right) f^{n-i}=0
$$

with $b_{i} \in A B\left(G \backslash E_{0}\right)$ for each $i$. Thus we may regard the functions $b_{1}, \ldots, b_{n}$ as defined all over $G$. But it is now a simple verification to show that on $f_{0}^{-1}(G)$

$$
f^{n}+\sum_{i=1}^{n}\left(c_{i} \circ f_{0}\right) f^{n-i}=0
$$

with $c_{i} \in M(G)$ for each $i$. We conclude that $c_{i}$ is an extension of $a_{i} \mid G \backslash E_{0}$ over $G \cap E_{0}, i=1, \ldots, n$. Thus, preserving the notation, we have the relation (1) valid on $f_{0}^{-1}(D)$ with $a_{i} \in M(D)$ for each $i$.

As in the proof of Theorem 3, it can be shown that

$$
P(z, w)=w^{n}+\sum_{i=1}^{n} a_{i}(z) w^{n-i}=(Q(z, w))^{n^{\prime}}
$$

where $Q(z, w)$ is an irreducible polynomial over $M(D)$ of degree $n / n^{\prime}$. We claim that this degree equals 1 , i.e. $n^{\prime}=n$. In order to prove this, define, as in the proof of Theorem 3, the mapping $\varphi: V_{0} \rightarrow \tilde{D}$, where $\tilde{D}$ denotes the Riemann surface of the relation $Q(z, w)=0, z \in D$. Further denote by $\varphi^{*}$ the continuous extension of $\varphi$ to $V_{0} \cup \beta_{V_{0}}$, and put $q_{0}=\varphi^{*}\left(p_{0}\right)$. Since $q_{0} \in c^{-1}\left(E_{0}\right)$ (cf. again the proof of Theorem 3$)$ and $c^{-1}\left(E_{0}\right)$ is totally disconnected, we can find a Jordan region $J$ in $\tilde{D}$ containing $q_{0}$ with $\partial J \cap c^{-1}\left(E_{0}\right)=\emptyset$ and a conformal homeomorphism $\psi$ : $J \rightarrow D$. Denote by $U$ the component of $\varphi^{-1}(J)$ with $p_{0} \in \beta_{U}$. Then $v_{\varphi \mid U}$ is bounded by $n^{\prime}$ as appears from the definition of $\varphi$ in view of the representation (2). Thus $(\psi \circ \varphi) \mid U$ belongs to $A B(U)$, and $v_{(\psi \circ \varphi) \mid U}$ is bounded by $n^{\prime}$. By the minimality of $n=n\left(f_{0}\right)$ we have $n^{\prime} \geqq n$, and hence $n^{\prime}=n$.

We conclude that, for a certain $g \in M(D)$, the pair $\left(f_{0}, f\right)$ annihilates the polynomial $Q(z, w)=w-g(z)$, i.e. $f(p)=g\left(f_{0}(p)\right)$ for all $p \in V_{0}$. 
The uniqueness of $g$ is a consequence of the openness of $f_{0}$. The latter assertion of the theorem follows immediately from the former by Theorem 2 .

The following corollary is immediate although nontrivial in itself.

Corollary. Let $W$ be a Riemann surface of class $\mathscr{M}_{B}$, and let $V$ be a subregion of $W$ with compact relative boundary. Then $M C(V)$ is a field.

Remark. As shown by Heins ([3, p. 298]), there exist parabolic Riemann surfaces (which automatically satisfy the absolute $A B$-maximum principle) such that no relatively noncompact subregion with compact boundary tolerates a meromorphic function of bounded valence. Thus for these surfaces the class $M C(W)$ reduces to that of constants, while the problem considered in Theorem 5 has no sense.

\section{Riemann surfaces with $M C=B V$}

2.1. Let $E$ be a proper closed subset of $\hat{\boldsymbol{C}}$. Then $E$ is said to be of class $N_{C}$ if, for every domain $G \subset \hat{\boldsymbol{C}}$ with $E \subset G$, every function $G \rightarrow C$ continuous on $G$ and analytic on $G \backslash E$ is actually analytic all over $G$. The subclass of $N_{C}$ constituted by the totally disconnected elements of $N_{C}$ is denoted by $N_{C}^{\prime}$. It is known that every closed set $E \subset \hat{C}$ of $\sigma$-finite linear measure is of class $N_{C}$ and, on the other hand, no set whose Hausdorff dimension exceeds 1 is of class $N_{C}$ (see e.g. [2, Chapter 3]). Clearly, $N_{B} \subseteq N_{C}^{\prime}$, and it can be shown that $N_{C}^{\prime}$ and $N_{D}$, as well as $N_{C}^{\prime}$ and $N_{S B}$, overlap.

Given any Riemann surface $W$, a closed totally disconnected subset $E$ of $W$ is said to be of class $N_{C}^{\prime}$ in $W$ if for every parametric disc $(V, \varphi)$ of $W$ the compact parts of $\varphi(E \cap V) \subset \hat{\boldsymbol{C}}$ are of class $N_{C}^{\prime}$. If, in particular, $W$ is a compact surface, one can find a planar subregion $V$ of $W$ such that $E \subset V$, and it is readily seen that $E$ is of class $N_{C}^{\prime}$ in $W$ if and only if $\psi(E) \subset \hat{\boldsymbol{C}}$ is of class $N_{C}^{\prime}$ for any conformal mapping $\psi: V \rightarrow \hat{C}$. In fact, it can be shown by a standard use of Cauchy's integral formula (cf. e.g. [1, p. 108]) that $E \subset \hat{C}$ is of class $N_{C}^{\prime}$ if and only if every point $z \in E$ has arbitrarily small neighborhoods $U_{z}$ such that $E \cap U_{z}$ are of class $N_{C}^{\prime}$.

We shall make use of the following lemma; the proof proceeds as in [5, Lemma 5] and will be omitted.

Lemma 2. Let $W$ be a Riemann surface, and let $f: W \rightarrow \hat{C}$ be a nonconstant analytic mapping.

(a) If $E \subset \hat{\boldsymbol{C}}$ is of class $N_{C}^{\prime}$, then $f^{-1}(E)$ is of class $N_{C}^{\prime}$ in $W$.

(b) If $E \subset W$ is a compact set of class $N_{C}^{\prime}$ in $W$, then $f(E)$ is of class $N_{C}^{\prime}$.

We shall need the following extension of Theorem 4 . 
Theorem 6. Let $W$ be a Riemann surface of class $\mathscr{D}\left(N_{C}^{\prime}\right)$. Then $M C(W)$ is a field, and there exists an analytic mapping $\varphi$ of $W$ into a compact Riemann surface $W^{\prime}$, uniquely determined up to a conformal equivalence, such that $g \mapsto g \circ \varphi$ is an isomorphism of $M\left(W^{\prime}\right)$ onto $M C(W)$. Moreover, we have $M C(W) \subset B V(W)$ and $D_{f} \in N_{C}^{\prime}$ for each nonconstant $f \in M C(W)$.

Proof. Let $f_{0}$ be a nonconstant $B V$-function on $W$ with $D_{f_{0}} \in N_{C}^{\prime}$, and denote by $n$ the constant valence of $f_{0} \mid W \backslash f_{0}^{-1}\left(D_{f_{0}}\right)$. Assume that $f \in M C(W)$ is nonconstant. We have on $W \backslash f_{0}^{-1}\left(D_{f_{0}}\right)$

$$
f^{n}+\sum_{i=1}^{n}\left(a_{i} \circ f_{0}\right) f^{n-i}=0,
$$

where $a_{1}, \ldots, a_{n} \in M\left(\hat{\boldsymbol{C}} \backslash D_{f_{0}}\right)$. We claim that $a_{i}$ admits a meromorphic extension to $D_{f_{0}}$, i.e. $a_{i}$ is a rational function on $\hat{\boldsymbol{C}}$ for each $i$.

So fix $z_{0} \in D_{f_{0}}$. Since $D_{f_{0}}$ is totally disconnected, $f_{0}$ admits a continuous extension $f_{0}^{*}$ over the ideal boundary $\beta$ with $f_{0}^{*}(\beta) \subset D_{f_{0}}$ (Lemma 1 ). As in the case of Theorem $5,\left(f_{0}^{*}\right)^{-1}\left(z_{0}\right)$ consists of at most $n$ points $p_{1}, \ldots, p_{k}$, and we may attach to each $p_{i} n\left(p_{i} ; f_{0}\right)$, the multiplicity of $f_{0}$ (see the definitions preceding Theorem 5). Further, we can find a linear fractional mapping $\psi$ such that $g=\left(\psi \circ f^{*}\right) \mid U$ is bounded for some neighborhood $U$ of $\left\{p_{1}, \ldots, p_{k}\right\}$. Let $G$ be a neighborhood of $z_{0}$ such that $\partial G \cap D_{f_{0}}=\emptyset$ and $\left(f_{0}^{*}\right)^{-1}(G) \subset U$. We have on $f_{0}^{-1}\left(G \backslash D_{f_{0}}\right)$

$$
g^{n}+\sum_{i=1}^{n}\left(b_{i} \circ f_{0}\right) g^{n-i}=0
$$

with $b_{i} \in A B\left(G \backslash D_{f_{0}}\right)$ for each $i$. Moreover, as $z \rightarrow z_{0}$ in $G \backslash D_{f_{0}}$, clearly $b_{1}(z) \rightarrow$ $\sum_{i=1}^{k} n\left(p_{i} ; f_{0}\right) g\left(p_{i}\right)$. Because $D_{f_{0}} \in N_{C}^{\prime}$, we conclude that $b_{1}$ admits an analytic extension over $G$. Since the same is readily seen to be true of $b_{2}, \ldots, b_{n}$ as well, we may regard them as defined and analytic all over $G$. It follows that

$$
f^{n}+\sum_{i=1}^{n}\left(c_{i} \circ f_{0}\right) f^{n-i}=0
$$

with $c_{i} \in M(G)$ for each $i$; this is valid, by continuity, on $f_{0}^{-1}(G)$. Thus $c_{i} \mid G \backslash D_{f_{0}}=$ $a_{i} \mid G \backslash D_{f_{0}}$ for each $i$. Altogether, the functions $a_{1}, \ldots, a_{n}$ are restrictions of rational functions on $\hat{\boldsymbol{C}}$, and $f$ is algebraic over $M_{0}(W)=\left\{g \in M(W) \mid g=a \circ f_{0}\right.$ for some $a \in M(\hat{\boldsymbol{C}})\}$, a subfield of $M(W)$.

Now suppose conversely that $f \in M(W)$ is nonconstant and algebraic over $M_{0}(W)$. Then we have on $W$

$$
f^{m}+\sum_{i=1}^{m}\left(b_{i} \circ f_{0}\right) f^{m-i}=0
$$

for some integer $m$ and with $b_{1}, \ldots, b_{m}$ in $M(\hat{\boldsymbol{C}})$. Suppose that $w_{0} \in \mathrm{C} 1(f ; \beta)$. Then there exists a sequence of points $\left(p_{n}\right)$ in $W$ such that $p_{n} \rightarrow p_{0}$ for some $p_{0} \in \beta$ and 
$f\left(p_{n}\right) \rightarrow w_{0}$. Set $z_{0}=f_{0}^{*}\left(p_{0}\right), z_{n}=f_{0}\left(p_{n}\right)$ and $w_{n}=f\left(p_{n}\right), n=1,2, \ldots$. We have

$$
w_{n}^{m}+\sum_{i=1}^{m} b_{i}\left(z_{n}\right) w_{n}^{m-i}=0
$$

for each $n$. Hence by continuity,

as well. We infer that

$$
w_{0}^{m}+\sum_{i=1}^{m} b_{i}\left(z_{0}\right) w_{0}^{m-i}=0
$$

$$
\mathrm{Cl}(f ; \beta) \subset\left\{w \in \hat{\boldsymbol{C}} \mid w^{m}+\sum_{i=1}^{m} b_{i}(z) w^{m-i}=0 \text { for some } z \in D_{f_{0}}\right\} .
$$

But since $D_{f_{0}}$ is closed and totally disconnected, the same is true of $\mathrm{C} 1(f ; \beta)$. Hence $f \in M C(W)$. Thus $M C(W)$ coincides with the subfield of $M(W)$ constituted by the algebraic elements over $M_{0}(W)$. More precisely, by resorting to the theorem of the primitive element, it is readily seen (cf. the proof of [5, Theorem 7]) that $M C(W)$ is a simple algebraic extension of $M_{0}(W)$.

Let $f_{1}$ be a primitive element of $M C(W)$, and let

$$
X^{m}+\sum_{i=1}^{m}\left(a_{i} \circ f_{0}\right) X^{m-i}, \quad a_{1}, \ldots, a_{m} \in M(\hat{\boldsymbol{C}}),
$$

be the minimal polynomial of $f_{1}$. Since

$$
P(z, w)=w^{m}+\sum_{i=1}^{m} a_{i}(z) w^{m-i}
$$

is irreducible, the Riemann surface $W^{\prime}$ of the relation $P(z, w)=0, z \in \hat{\boldsymbol{C}}$, is compact and connected. Define the mapping $\varphi: W \rightarrow W^{\prime}$ as in the proof of Theorem 3; we have $f_{0}=c \circ \varphi, f_{1}=v \circ \varphi, c$ or $v$ being the center mapping or the value mapping, respectively. It is readily seen that $g \mapsto g \circ \varphi$ is an isomorphism of $M\left(W^{\prime}\right)$ onto $M C(W)$; for the details as well as for the proof of the uniqueness of $W^{\prime}$ we refer to $[5$, pp. 21-22].

To prove the last assertion of the theorem, take a nonconstant $f \in M C(W)$ and choose $g \in M\left(W^{\prime}\right)$ such that $f=g \circ \varphi$. Since $\varphi \mid W \backslash \varphi^{-1}\left(c^{-1}\left(D_{f_{0}}\right)\right)=$ $\varphi \mid W \backslash f_{0}^{-1}\left(D_{f_{0}}\right)$ has constant valence $n / m$ on $W^{\prime} \backslash c^{-1}\left(D_{f_{0}}\right)$, we have immediately $f \in B V(W)$. Thus $M C(W) \subset B V(W)$. Moreover, if $w_{0} \in \hat{C} \backslash g\left(c^{-1}\left(D_{f_{0}}\right)\right)$, then $v_{f}\left(w_{0}\right)=\max \left\{v_{f}(w) \mid w \in \hat{C}\right\}$. But $g\left(c^{-1}\left(D_{f_{0}}\right)\right)$ is of class $N_{C}^{\prime}$ by Lemma 2. The proof is complete.

Remark. The above result is sharp, i.e. the class $N_{C}^{\prime}$ cannot be replaced by a larger one, provided we insist on total disconnectedness (cf. Lemma 3 and Lemma 4 below). The inclusion $M C(W) \subset B V(W)$ is strict, in general. To provide an example, let $E$ be a closed totally disconnected subset of the unit circle $C=\{z \in C|| z \mid=1\}$ such that the inner capacity of $C \backslash E$ is $<1$ (see [1, p. 124]). Then $\hat{\boldsymbol{C}} \backslash E$ carries 
nonrational univalent (meromorphic) functions ([1, Theorem 6 and Theorem 14]) while $M C(\hat{\boldsymbol{C}} \backslash E)=M(\hat{\boldsymbol{C}})$.

Let us state the following local counterparts of the preceding theorem. The proofs will be omitted, for all ingredients needed can be found in the previous considerations (cf. in particular Theorem 3 and Theorem 5).

Theorem 7. Let $W$ be a Riemann surface of class $\mathscr{D}\left(N_{C}^{\prime}\right)$, and let $V$ be a subregion of $W$ with compact boundary. Then every nonconstant function $f \in M C(\bar{V})$ has bounded valence, and $\mathrm{C} 1\left(f ; \beta_{V}\right)=f^{*}\left(\beta_{V}\right) \in N_{C}^{\prime}$.

Theorem 8. Let $W$ be a Riemann surface of class $\mathscr{D}\left(N_{C}^{\prime}\right)$, and suppose that $p$ belongs to the ideal boundary of $W$. Then there exist a subregion $V$ of $W$ with compact $\partial V$ and with $p \in \beta_{V}$ and an analytic function $f_{0}: V \rightarrow D=\{z \in \hat{\boldsymbol{C}}|| z \mid<1\}$ such that, given any $f \in M C(V)$, one can find a unique $g \in M(D)$ satisfying $f=g \circ f_{0}$.

2.2. We are now in a position to establish our main result.

The orem 9. Let $W$ be a Riemann surface of class $\mathscr{D}\left(N_{C} \cap N_{S B}\right)$. Then $M C(W)=$ $B V(W)$. The class $N_{C} \cap N_{S B}$ cannot, without further restrictions, be replaced by a larger one; in fact, for a Riemann surface $W$ of finite genus we have $M C(W)=$ $B V(W)$ only if $W \in \mathscr{D}\left(N_{C} \cap N_{S B}\right)$.

Proof. Let $f_{0}$ be a meromorphic function of bounded valence on $W$ such that $D_{f_{0}} \in N_{C} \cap N_{S B}$. Since sets of class $N_{S B}$ are totally disconnected, we have even $D_{f_{0}} \in N_{C}^{\prime}$. Thus Theorem 6 applies, and we infer that $M C(W) \subset B V(W)$.

On the other hand, it follows from the assumption that $D_{f_{0}}$ is even of class $N_{D}$, for in actual fact $N_{C} \cap N_{S_{B}}=N_{C} \cap N_{D}$. Indeed, let $\varphi$ be any univalent meromorphic function on $\hat{\boldsymbol{C}} \backslash D_{f_{0}}$. Since $D_{f_{0}} \in N_{S B}, \hat{\boldsymbol{C}} \backslash \varphi\left(\hat{\boldsymbol{C}} \backslash D_{f_{0}}\right)$ also must be of class $N_{S B}$; in particular, $\hat{\boldsymbol{C}} \backslash \varphi\left(\hat{\boldsymbol{C}} \backslash D_{f_{0}}\right)$ is totally disconnected. Thus $\varphi$ admits a topological extension $\varphi^{*}$ to the whole sphere. But the relation $D_{f_{0}} \in N_{C}$ implies that $\varphi^{*}$ is conformal on $\hat{\boldsymbol{C}}$. The assertion now follows from [1, Theorem 6]. Accordingly, we may apply [5, Theorem 7]. It follows, in particular, that $D_{f}$ is totally disconnected for every nonconstant $f \in B V(W)$. We conclude from Lemma 1 that $B V(W) \subset M C(W)$.

In order to prove the necessity of the condition $W \in \mathscr{D}\left(N_{C} \cap N_{S B}\right)$ for surfaces of finite genus, we need some auxiliary results.

Lemma 3. Let $W$ be an open Riemann surface, let $\beta$ be the ideal boundary of $W$, and let $f \in A C(W)$. Then $f^{*}(\beta)=f^{*}(W \cup \beta)$. Moreover, if $f \in A C(W)$ is nonconstant, then the set $\left\{z \in \hat{\boldsymbol{C}} \mid v_{f}(z)=\infty\right\}$ is residual in $f^{*}(\beta)$.

Proof. Suppose that $f \in A C(W)$ is nonconstant, and assume that there exists a point $p_{0} \in W$ such that $f\left(p_{0}\right) \notin f^{*}(\beta)$. Let $d$ denote the mutual distance of $\left\{f\left(p_{0}\right)\right\}$ and $f^{*}(\beta)$. Choose then, for every $p \in \beta$, an open neighborhood $U_{p}$ such that $\partial U_{p}$, 
the relative boundary of $U_{p}$, is compact and $f^{*}\left(U_{p}\right) \subset D\left(f^{*}(p), d / 2\right)=$ $\left\{z \in C|| z-f^{*}(p) \mid<d / 2\right\}$. From the open covering $\left\{U_{p} \mid p \in \beta\right\}$ of $\beta$ pick out a finite subcovering $\left\{U_{p_{1}}, \ldots, U_{p_{k}}\right\}$. Let $\left(V_{n}\right)$ be a standard exhaustion of $W$ (in particular, the components of $W \backslash V_{n}$ are noncompact). Since $F=\bigcup_{i=1}^{k} \partial U_{p_{i}}$ is a compact subset of $W$, there exists a positive integer $n_{0}$ such that $F \cup\left\{p_{0}\right\} \subset V_{n}$ for $n \geqq n_{0}$. A moment's thought then reveals that for every component $C$ of $W \backslash V_{n_{0}}$ there is $i \in\{1, \ldots, k\}$ such that $C \subset U_{p_{i}}$. Let $B_{1}, \ldots, B_{m}$ be the components of $\partial V_{n_{0}}$. We infer that each $f\left(B_{i}\right)$ is contained in some $D\left(f^{*}\left(p_{j}\right), d / 2\right), j=1, \ldots, k$. Thus the winding number of $f\left(B_{i}\right)$ with respect to $f\left(p_{0}\right)$ is 0 for each $i$. We conclude from the argument principle that $p_{0} \notin f\left(V_{n_{0}}\right)$. This is a contradiction. Hence $f(W) \subset f^{*}(\beta)$.

To prove the latter statement, let again $\left(V_{n}\right)$ be an exhaustion of $W$, and denote by $F_{n}$ the closed set $f^{*}(\beta) \backslash f\left(W \backslash \bar{V}_{n}\right), n=1,2, \ldots$ By continuity, $f^{*}(\beta)=$ $\overline{f\left(W \backslash \bar{V}_{n}\right)}$, so that $F_{n}$ is a nowhere dense subset of $f^{*}(\beta)$ for each $n$. But clearly $f^{*}(\beta) \backslash \bigcup_{n=1}^{\infty} F_{n} \subset\left\{z \in \hat{\boldsymbol{C}} \mid v_{f}(z)=\infty\right\}$. The assertion follows.

Lemma 4. Let $W$ be a compact Riemann surface, and let $E$ be a closed totally disconnected subset of $W$ failing to be of class $N_{C}^{\prime}$ in $W$. Then the class $A C(W \backslash E)$ contains a nonconstant function.

Proof. We may assume that $E$ contains no inessential points, i.e. for every parametric disc $(V, \varphi)$ of $W$ with $V \cap E \neq \emptyset$ and $\partial V \cap E=\emptyset$ the set $\varphi(V \cap E)$ fails to be of class $N_{C}$. Let $g$ denote the genus of $W$. Since $E$ is totally disconnected, we can find (cf. [13, p. 262]) planar regions $U_{1}, \ldots, U_{2 g+1} \subset W$ such that $\bar{U}_{i} \cap \bar{U}_{j}=\emptyset$ if $i \neq j, E_{i}=U_{i} \cap E \neq \emptyset$ and $\partial U_{i} \cap E=\emptyset$ for each $i$. We may further assume that $U_{i}$ is simply connected and that there exists a nonanalytic continuous function $f_{i}: U_{i} \rightarrow \boldsymbol{C}$ which is analytic on $U_{i} \backslash E_{i}, i=1, \ldots, 2 g+1$.

Consider the harmonic function $\operatorname{Re} f_{i}$ on $U_{i} \backslash E_{i}$. Clearly,

$$
\int_{\gamma} * d\left(\operatorname{Re} f_{i}\right)=0
$$

for every cyle $\gamma$ in $U_{i} \backslash E_{i}$. Thus, by [11, Theorem 3], there exists a real harmonic function $v_{i}$ on $W \backslash E_{i}$ such that $\operatorname{Re} f_{i}-v_{i}$ has a harmonic extension to $U_{i}$. It is readily seen that $* d v_{i}$ has a vanishing period along every dividing cycle in $W \backslash E$. Let $\gamma_{k}, k=1, \ldots, 2 g$, be $2 g$ nondividing canonical cycles of $W \backslash E$, and consider the system of equations

$$
\sum_{i=1}^{2 g+1} c_{i}\left(\int_{\gamma_{k}} * d v_{i}\right)=0 \quad(k=1, \ldots, 2 g) .
$$

Pick out a nontrivial solution $\left(c_{1}, \ldots, c_{2 g+1}\right) \neq(0, \ldots, 0)$. Since the differential $* d\left(\sum_{i=1}^{2 g+1} c_{i} v_{i}\right)$ has a vanishing period along every cycle in $W \backslash E$, there exists an analytic function $f$ on $W \backslash E$ with $\operatorname{Re} f=\sum_{i=1}^{2 g+1} c_{i} v_{i}$. 
We proceed to show that $f$ admits a continuous extension over $E$. Fix $j \in\{1, \ldots, 2 g+1\}$, and denote by $u_{j}: U_{j} \rightarrow C$ the harmonic extension of $\operatorname{Re} f_{j}-v_{j}$ over $E_{j}$. Let $u_{j}^{*}$ denote a conjugate harmonic function of $u_{j}$ on $U_{j}$, and let $v_{i}^{*}$ denote a conjugate harmonic function of $v_{i} \mid U_{j}, i \neq j$. Further denote by $h_{j}$ the analytic function $-c_{j}\left(u_{j}+i u_{j}^{*}\right)+\sum_{i \neq j} c_{i}\left(v_{i}+i v_{i}^{*}\right)$ on $U_{j}$. Then $\operatorname{Re} f-\operatorname{Re} h_{j}=c_{j}\left(u_{j}+v_{j}\right)=$ $\operatorname{Re} c_{j} f_{j}$ on $U_{j} \backslash E_{j}$. Hence there exists a constant $a_{j}$ such that $f=h_{j}+c_{j} f_{j}+a_{j}$ on $U_{j} \backslash E_{j}$. We may now define

$$
f^{*}(p)=h_{j}(p)+c_{j} f_{j}(p)+a_{j} \text { for } p \in E_{j} .
$$

This representation permits us also to conclude that $f$ is nonconstant. Indeed, let $j$ be chosen such that $c_{j} \neq 0$. Then $c_{j} f_{j}$ is nonanalytic while $h_{j}+a_{j}$ is analytic on $U_{j}$. Hence $f$ cannot be constant.

The following lemma can be regarded as a natural generalization of [1, Theorem 6].

Lemma 5. Let $W$ be a compact Riemann surface, and let $E$ be a proper closed subset of $W$ such that $W \backslash E$ is connected. Then $E$ is of class $N_{D}$ in $W$ if and only if every meromorphic function of bounded valence on $W \backslash E$ is the restriction to $W \backslash E$ of a rational function on $W$.

Proof. Suppose first that $E \subset W$ is of class $N_{D}$ in $W$. Let $f \in B V(W \backslash E)$. It follows from [5, Lemma 6] that $f$ admits a meromorphic extension to $W$, i.e. $f$ is the restriction of a rational function on $W$.

Suppose conversely that $E$ fails to be of class $N_{D}$ in $W$. If the interior of $E$ is nonempty, we can find a nonconstant function $f \in M(W)$ such that $f \mid W \backslash E$ is bounded. If $g$ is a nonalgebraic univalent function on $f(W \backslash E)$, then $(g \circ f) \mid W \backslash E$ is clearly nonrational and belongs to $B V(W \backslash E)$. Assume now that $E$ is nowhere dense in $W$ and fails to be of class $N_{S B}$. By [6, Theorem 12] and [13, Theorem X. 3 C] $W \backslash E$ is essentially extendable, i.e. there exist a compact Riemann surface $W^{*}$ and a closed subset $F$ of $W^{*}$ with nonempty interior such that $W \backslash E$ is conformally equivalent to $W^{*} \backslash F$. Hence we can again find a nonconstant bounded function in $B V(W \backslash E)$. However, for any $f \in M(W), f \mid W \backslash E$ is unbounded, because $f(E)$ is nowhere dense in $\hat{\boldsymbol{C}}$.

There remains the case $E \in N_{S B} \backslash N_{D}$ in $W$. It follows from [8, Theorem 1] and [12, Theorem I. $8 \mathrm{E}$ ] that $W \backslash E$ then admits conformally nonequivalent closed extensions. Hence we can find a compact Riemann surface $W^{*}$, a closed subset $F$ of $W^{*}$ and a conformal homeomorphism $\varphi: W \backslash E \rightarrow W^{*} \backslash F$ which, by [6, Theorem 12], extends to a nonconformal homeomorphism $\varphi^{*}: W \rightarrow W^{*}$. Let $g$ be a nonconstant function in $M\left(W^{*}\right)$. We claim that $f=g \circ \varphi$ does not admit a meromorphic extension to $W$. Assume this is not the case, and denote by $f^{*}$ the extended function. Then we have, by continuity, $f^{*}=g \circ \varphi^{*}$. Let $B_{g}$ denote 
the finite set of zeros of the derivative of $g$, and let $p \in W \backslash\left(f^{*}\right)^{-1}\left(g\left(B_{q}\right)\right)$. Choose a neighborhood $U_{p}$ of $p$ such that $g^{-1}$ is defined on $f^{*}\left(U_{p}\right)$ (the branch of $g^{-1}$ is chosen in such a way that $\left.g^{-1}\left(f^{*}(p)\right)=\varphi^{*}(p)\right)$. Thus we have $\varphi^{*}(q)=\left(g^{-1} \circ f^{*}\right)(q)$ for $q \in U_{p}$. Consequently, $\varphi^{*}$ is analytic on $W \backslash\left(f^{*}\right)^{-1}\left(g\left(B_{g}\right)\right)$ and, by the discreteness of $\left(f^{*}\right)^{-1}\left(g\left(B_{g}\right)\right)$, all over $W$. This contradiction completes the proof.

We return to the proof of Theorem 9. So let $W$ be a Riemann surface of finite genus. We may assume that $W$ is a subregion of a compact Riemann surface $W^{*}$. Let $E$ denote the set $W^{*} \backslash W$. Suppose first that some component of $E$ is nondegenerate. As in the proof of Lemma 5, we can find a bounded nonconstant function in $B V(W)$. But Lemma 3 implies that such a function cannot belong to the class $M C(W)$. Thus $M C(W) \neq B V(W)$ in this case.

Now suppose that $E$ is totally disconnected but fails to be of class $N_{C}^{\prime}$ in $W^{*}$. By Lemma 4 there exists a nonconstant function $f$ in $A C(W)$, and by Lemma 3 $f \notin B V(W)$. We have again $M C(W) \neq B V(W)$.

Then suppose that $E \in N_{C}^{\prime} \backslash N_{D}$ in $W^{*}$. By Lemma $5, \quad B V(W) \backslash M\left(W^{*}\right) \neq \emptyset$. But clearly $M C(W)=M\left(W^{*}\right)$. Hence $M C(W) \neq B V(W)$.

There remains the case $E \in N_{C}^{\prime} \cap N_{D}$ (then, of course, $M C(W)=B V(W)=$ $\left.M\left(W^{*}\right)\right)$. Let $f_{0}$ be a nonconstant function in $M\left(W^{*}\right)$. It follows from [5, Lemma 5] and Lemma 2 that $f_{0}(E) \in N_{C} \cap N_{D}=N_{C} \cap N_{S B}$. Thus $D_{f_{0} \mid W} \in N_{C} \cap N_{S B}$, i.e. $W \in \mathscr{D}\left(N_{C} \cap N_{S B}\right)$. The proof is complete.

Remark. In view of Theorem 2, one may ask how the class $M D^{*}(W)$ is related to $M C(W)$ in the case $W \in \mathscr{D}\left(N_{C} \cap N_{S B}\right)$. Of course $M C(W) \subset M D^{*}(W)$, but we do not know whether the opposite inclusion also holds. However, under the additional hypothesis on $f \in M D^{*}(W)$ that $\left\{z \in \hat{\boldsymbol{C}} \mid v_{f}(z)<\infty\right\}$ be of second category, it can be shown that $f \in M C(W)$.

\section{References}

[1] Ahlfors, L., and A. Beurling: Conformal invariants and function-theoretic null-sets. - Acta Math. 83, 1950, 101-129.

[2] Garnetr, J.: Analytic capacity and measure. - Lecture Notes in Mathematics 297, SpringerVerlag, Berlin-Heidelberg-New York, 1972.

[3] Heins, M.: Riemann surfaces of infinite genus. - Ann. of Math. 55, 1952, 296-317.

[4] HeIns, M.: On certain meromorphic functions of bounded valence. - Rev. Roumaine Math. Pures Appl. 2, 1957, 263-267.

[5] JäRVI, P.: Removability theorems for meromorphic functions. - Ann. Acad. Sci. Fenn. Ser. A I Math. Dissertationes 12, 1977, 1-33.

[6] JuRchescu, M.: Modulus of a boundary component. - Pacific J. Math. 8, 1958, 791-809.

[7] Myrberg, L.: Über quasirationale Funktionen auf parabolischen Riemannschen Flächen. Ann. Acad. Sci. Fenn. Ser. A I 404, 1967, 1-33.

[8] OIKAWA, K.: On the uniqueness of the prolongation of an open Riemann surface of finite genus. - Proc. Amer. Math. Soc. 11, 1960, 785-787. 
[9] Ozawa, M.: Meromorphic functions on certain Riemann surfaces. - Ibid. 16, 1965, 747-751.

[10] Royden, H. L.: Riemann surfaces with the absolute AB-maximum principle. - Proceedings of the Conference on Complex Analysis, Minneapolis, 1964, edited by A. Aeppli, E. Calabi and H. Röhrl, Springer-Verlag, Berlin-Heidelberg-New York, 1965, $172-175$.

[11] Royden, H. L.: Function theory on compact Riemann surfaces. - J. Analyse Math. 18. 1967, 295-327.

[12] SARIo, L., and M. NAKAI: Classification theory of Riemann surfaces. - Die Grundlehren der mathematischen Wissenschaften 164, Springer-Verlag, Berlin-Heidelberg-New York, 1970.

[13] SARio, L., and K. OiKawa: Capacity functions. - Die Grundlehren der mathematischen Wissenschaften 149, Springer-Verlag, Berlin-Heidelberg-New York, 1969.

University of Helsinki

Department of Mathematics

SF-00100 Helsinki 10

Finland

Received 20 September 1979

Revision received 23 January 1980 